EPJ Web of Conferences 113, 08016 (2016)

DOI: $10.1051 /$ epjconf/201611308016

(C) Owned by the authors, published by EDP Sciences, 2016

\title{
The Breakup Cross Section of the D+D Reaction at 6.94 MeV
}

\author{
A.L. Richard ${ }^{1}$, a , C.R. Brune ${ }^{1}$, D.C. Ingram ${ }^{1}$, S. Dhakal ${ }^{1}$, A. Karki², \\ T.N. Massey ${ }^{1}$, J.E. O'Donnell ${ }^{1, b}$, and C.E. Parker ${ }^{1}$ \\ ${ }^{1}$ Ohio University, Athens, $\mathrm{OH} 45701$, USA \\ ${ }^{2}$ University of Vermont, Burlington, VT 05405, USA
}

\begin{abstract}
The D+D reactions are well known and widely used for a variety of purposes, mainly because of the use of the $\mathrm{D}(d, n)^{3} \mathrm{He}$ reaction as a mono-energetic neutron source. The least studied of the $\mathrm{D}+\mathrm{D}$ reactions is the $\mathrm{D}(d, n) \mathrm{pD}$ reaction known as the deuteron breakup reaction, which produces a continuum of neutrons at energies below the monoenergetic peak. The neutron energy distribution as a function of angle for the cross section, $\frac{d^{2} \sigma}{d \Omega d E}$, of the $\mathrm{D}(d, n) \mathrm{pD}$ reaction has been measured using a $6.94-\mathrm{MeV}$ pulsed deuteron beam incident upon a $\mathrm{D}_{2}$ gas target. The time-of-flight technique was used to determine the energy of the neutrons detected in an array of two lithium glass scintillators and one NE-213 scintillator. The breakup cross section was determined as low as $225-\mathrm{keV}$ neutron energy in the lithium glass detectors.
\end{abstract}

\section{Introduction and Experiment}

The $\mathrm{D}+\mathrm{D}$ reactions yield mono-energetic neutrons from the $\mathrm{D}(d, n)^{3} \mathrm{He}$ reaction and a continuum of low-energy neutrons from the $\mathrm{D}(d, n) \mathrm{pD}$ reaction for $7-\mathrm{MeV}$ incident deuterons. The $\mathrm{D}(d, n) \mathrm{pD}$ reaction occurs when the incident deuteron energy is above the threshold of $4.45 \mathrm{MeV}$, causing one of the deuterons to break up into its constituent particles, the proton and the neutron. This reaction produces a continuum of neutrons at energies lower than the mono-energetic neutrons from the $\mathrm{D}(d, n)^{3} \mathrm{He}$ reaction.

In this work, the $\mathrm{D}(d, n) \mathrm{pD}$ reaction has been studied for the purpose of use as a neutron source for the active interrogation of hidden fissile materials. The $\mathrm{D}+\mathrm{D}$ reaction yields mono-energetic neutrons up to approximately $8 \mathrm{MeV}$ based upon the incident deuteron energy and yields breakup neutrons at lower energies. The $\mathrm{D}+\mathrm{D}$ reaction, as a neutron source, is attractive because unlike mono-energetic neutron sources that use radioactive materials, such as tritium, special regulations are not required. Neutrons from the $\mathrm{D}(d, n)^{3} \mathrm{He}$ reaction have been fully characterized, but neutrons resulting from the breakup reaction have not received this level of attention. Prior to this work, the $\mathrm{D}(d, n) \mathrm{pD}$ cross section was not measured below a neutron energy of $1.5 \mathrm{MeV}$, where a substantial contribution to the cross section occurs.

\footnotetext{
a e-mail: ar286106@ohio.edu

$\mathrm{b}_{\text {deceased }}$
} 


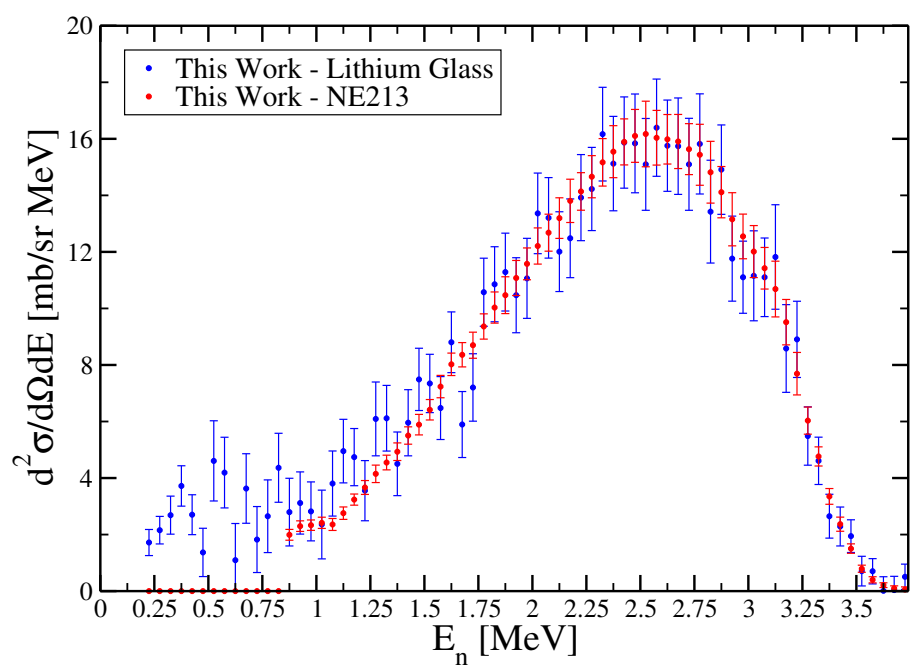

Figure 1. The measured neutron energy distribution of the cross section of the $\mathrm{D}(d, n) \mathrm{pD}$ reaction at $0^{\circ}$ for an incident deuteron energy of $6.94 \mathrm{MeV}$. The data were measured using NE-213 and lithium glass detectors.

\section{Results and Discussion}

The neutron energy distribution as a function of angle for the cross section, $\frac{d^{2} \sigma}{d \Omega d E}$, of the $\mathrm{D}(d, n) \mathrm{pD}$ reaction has been measured at the Edwards Accelerator Laboratory of Ohio University, using a 6.94$\mathrm{MeV}$ pulsed deuteron beam incident upon a $\mathrm{D}_{2}$ gas target. Data for the cross section range between laboratory angles of $0^{\circ}$ to $60^{\circ}$, in $5^{\circ}$ increments. The $0^{\circ}$ distribution is shown in Fig. 1 . The timeof-flight technique was used to determine the energy of the neutrons detected in an array consisting of two lithium glass scintillators and one NE-213 scintillator. The NE-213 scintillator has a good neutron detection efficiency over a wide energy range and is practical for use down down to about $1 \mathrm{MeV}$. Lithium glass scintillators have a non-zero efficiency below $1 \mathrm{MeV}$, and were utilized to detect continuum neutrons from the breakup reaction down to $225 \mathrm{keV}$.

The breakup cross section was determined for neutron energies as low as $225 \mathrm{keV}$ in the lithium glass detectors, well below the previous limits. Preliminary results from this work can be found in [1]. Simultaneous fits to the $\frac{d^{2} \sigma}{d \Omega d E}$ to test the self-consistency of the data are underway, which will allow for extrapolating cross section results to other laboratory angles and neutron energies as well as interpolating between existing results.

\section{Acknowledgements}

This project was funded in part by the Defense Threat Reduction Agency (DTRA) through grant number HDTRA1-09-1-0059 and U.S. D.O.E. (NNSA) through grant number DE-NA0001837. The authors would like to thank D.K. Jacobs and D.E. Carter for their assistance with the setup and operation of the accelerator and electronics.

\section{References}

[1] A.L. Richard, Master's thesis, Ohio University, Athens, Ohio, 2014. 